\title{
Role of Perfusion CT in Assessing Tumor Blood Flow and Malignancy Level of Gastric Cancer
}

\author{
Asami Satoh $^{\mathrm{a}}$ Kiyohiko Shuto $^{\mathrm{a}}$ Shinichi Okazumi ${ }^{\mathrm{a}}$ Gaku Ohira ${ }^{\mathrm{a}}$ \\ Toshiyuki Natsume $^{a}$ Koichi Hayano ${ }^{a}$ Kazuo Narushima ${ }^{a}$ Hiroshige Saito $^{a}$ \\ Takumi Ohta $^{a}$ Yoshihiro Nabeya $^{a}$ Noriyuki Yanagawa ${ }^{b}$ Hisahiro Matsubara ${ }^{a}$ \\ ${ }^{a}$ Department of Frontier Surgery, Chiba University Graduate School of Medicine, and ${ }^{b}$ Department of Radiological \\ Technology, Chiba University Hospital, Chiba, Japan
}

\section{Key Words}

Perfusion computed tomography $\cdot$ Blood flow $\cdot$ Tumor

stroma $\cdot$ Angiogenesis $\cdot$ Intratumoral hemodynamics

\begin{abstract}
Background/Aims: Intratumoral hemodynamics or tumor perfusion is useful in understanding the pathological background of the cancer. A parameter for a non-invasive, preoperative assessment of tumor perfusion has yet to be developed. Methods: The study included 50 patients who underwent surgery for gastric cancer. Perfusion computed tomography (P-CT) was performed using a 16-row multidetector CT, and tumor blood flow ( $\mathrm{ml} / \mathrm{min} / 100 \mathrm{~g}$ tissue) values were measured. We compared blood flow with histopathological characteristics and evaluated its correlation with microvessel density and tumor stromal density and calculated the ratio of vessels and stromal tissue. Results: There was a significant decrease in blood flow in advanced tumor depth, peritoneal dissemination and undifferentiated subtypes. Cases with Lauren's diffuse type carcinoma were found to have decreased blood flow compared to the mixed or intestinal type. As for the stromal structure, despite the lack of correlation with microvessel density, blood flow significantly decreased with increased stromal density. Conclusions:
\end{abstract}

Decreased blood flow value acquired from P-CT may reflect a progressive state of gastric cancer. The pathological background for this relation involves the tumor stroma. Tumor perfusion decreased as the stage and malignant character of the tumor advanced, and therefore P-CT could be a better strategy to estimate the malignancy level of cancer.

Copyright $\odot 2010$ S. Karger AG, Basel

\section{Introduction}

Gastric cancer is one of the most common malignancies worldwide and although diagnosed at a high frequency, it is still characterized by a poor prognosis [1-3]. A curative resection is the best treatment method to achieve cure in gastric cancer, but less than half are expected to survive for more than 5 years [4]. It is important to identify those cases at significant risk for recurrence and poor prognosis that could be treated with adjuvant therapy. The need for more reliable prognostic factors for gastric cancer has resulted in reports on new methods to predict the patient outcome, but the classification based on stage is still the mainstream parameter of choice.

The assessment of tumor perfusion and hemodynamic changes is useful in understanding the pathological

\section{KARGER}

Fax +41613061234 E-Mail karger@karger.ch www.karger.com
(C) 2010 S. Karger AG, Basel

$0253-4886 / 10 / 0274-0253 \$ 26.00 / 0$

Accessible online at:

www.karger.com/dsu
Asami Satoh, MD

Department of Frontier Surgery, Chiba University Graduate School of Medicine

1-8-1 Inohana, Chuo-ku, Chiba City, Chiba 260-8677 (Japan)

Tel. +81 43226 2110, Fax +81 432262113

E-Mailsamasami@goo.jp 
background of the cancer and determining prognosis. Malignant tumor growth and metastasis require a constant new blood supply and tumor-associated angiogenesis plays a critical role in development and spread of malignant tumors [5]. Clinical trials have succeeded in improving the patient survival with various anti-angiogenesis agents $[6,7]$. In addition, intratumoral neovascularization is a significant prognostic factor in some types of cancers, such as breast, colorectal and lung cancer [8-10].

Preoperative gastric histopathology has almost always been diagnosed using conventional endoscopic specimens. These are, however, inadequate for assessing angiogenesis because of intratumor heterogeneity and microvessel density count using immunohistological staining of surgical specimens is currently the most popular marker to quantify angiogenesis. The evaluation of tumor perfusion with pathological specimens is not always possible, such as in cases where the tumor is unresectable. A parameter for a non-invasive, preoperative assessment of tumor angiogenesis has yet to be developed.

Perfusion computed tomography (P-CT) is a relatively new technology that allows measurement of physiological parameters associated with tumor perfusion. The regional map of perfusion constructed by P-CT color codes the heterogeneous perfusion of the whole tumor. Multiple reports on correlations between P-CT parameters and histological measurements of angiogenesis such as microvessel density show the use of P-CT as a marker of angiogenesis [11-13]. The main hemodynamic parameter is the tumor blood flow and the blood flow value was compared among 50 cases of gastric cancer.

The purpose of this study was to estimate the tumor perfusion in advanced gastric cancers and its relationship to the clinicopathological features of the tumor. Furthermore, to clarify the pathological background of tumor perfusion, the tumor stroma was evaluated along with microvessel density, and these values were compared with the tumor blood flow.

\section{Materials and Methods}

\section{Patient Population}

The study was approved by the ethics committee and informed consent was obtained from all patients. Patients were included in this study if they had histopathologically proven locally advanced gastric cancer, either with surgical or endoscopic specimens and underwent P-CT prior to surgical treatment. Patients were excluded from the study if the tumor was stage Ia, or if the lesion was undetectable on CT. Patients with an inadequate renal func- tion (serum creatinine level $>2.0 \mathrm{mg} / \mathrm{dl}$ ) were also excluded from the study. Between June 2005 and December 2007, 50 consecutive patients who met the criteria were enrolled. The study included 29 men and 21 women (median age 64.0 years, age range $31-86$ years).

\section{Surgical Treatment}

A total or distal gastrectomy with regional lymphadenectomy was performed in 40 patients. In the remaining 10 cases, the tumors were unresectable due to invasion of surrounding tissues (8 cases) and/or peritoneal dissemination (5 cases) and biopsy specimens from preoperative endoscopy were used for the pathological analysis. The pathological tumor stage was assessed using the tumor node metastasis (TNM) system according to the International Union against Cancer (UICC) [14]. Patients were classified as stage Ib in 5 , stage II in 8 , stage IIIa or b in 12 , and stage IV in 25 . Out of the 25 patients, 21 were categorized as stage IV for their M1 status. All of these patients possessed peritoneal dissemination, and 2 had metastases to the liver.

\section{P-CT Imaging Protocol}

Prior to the examination, a 20-gauge venous cannula was placed in the forearm and $20 \mathrm{mg}$ of spasmolytic hyoscine N-butylbromide (Buscopan; Boehringer Ingelheim, Germany) was administered intravenously, unless contraindicated. All patients, with an exception of patients with tumors too large and difficult for even water to pass, were given one unit dose of effervescent agents immediately before the CT scan to distend the stomach wall. A restraining strap was also placed around the patient's abdomen to limit movement.

P-CT was performed with a 16-row multidetector CT scanner (Lightspeed Ultra 16; GE Medical Systems, Milwaukee, Wisc., USA). For initial localization of the tumor, a non-enhanced scan of the upper abdomen was obtained without contrast medium during a breath hold at the end of inhalation. After localization, a 2 -cm tumor region (four sequential 5-mm slices) with the largest tumor diameter was selected by the author. A dynamic study of the selected area was performed in a single 40 -s breath hold at the end of inhalation at a static table position. Images were obtained $10 \mathrm{~s}$ after an intravenous injection of $40 \mathrm{ml}$ of Iomeprol (Iomeron 300; Bracco Diagnostics, Princeton, N.J., USA) containing $300 \mathrm{mg}$ iodine $/ \mathrm{ml}$ was given at a rate of $5 \mathrm{ml} / \mathrm{s}$ followed by $40 \mathrm{ml}$ of saline chaser with a dual power injector. The scanning parameters were as follows: $5-\mathrm{mm}$ reconstructed section thickness, $120 \mathrm{kVp}, 60$ $\mathrm{mA}$. After completion of the perfusion scans, intravenous contrast was administered at $3 \mathrm{ml} / \mathrm{s}$ and a routine abdominal study was obtained by using $1.25-\mathrm{mm}$ contiguous sections (1-s gantry rotation time, $120 \mathrm{kVp}, 60 \mathrm{~mA}$ ).

\section{Image Analysis}

The data were transferred to an image-processing workstation (Advantage Workstation 4.2, GE Medical Systems) and analyzed by using commercial deconvolution-based CT perfusion software (Functool 2.6; GE Medical Systems) and the body tumor program. The analysis is based on the following principles:

Contrast material injected as a rapid bolus fills the entire capillary network and arrives at equilibrium and then washes out from the network of the tissue. When iodinated contrast material can be considered as a purely intravascular tracer on dynamic CT, the concentration of the contrast material within tissue $\mathrm{Q}(\mathrm{t})$ can 
be obtained with the arterial input $\mathrm{Ca}(\mathrm{t})$. The change in contrast material volume is shown as the impulse residue function $\mathrm{R}(\mathrm{t})$ and when $\mathrm{F}$ is constant in time, the next equation comprises: $\mathrm{Q}(\mathrm{t})=$ $\mathrm{F} \cdot \mathrm{Ca}(\mathrm{t})^{*} \mathrm{R}(\mathrm{t})$, where ${ }^{*}$ is the convolution operator.

The $\mathrm{Q}(\mathrm{t})$ and $\mathrm{Ca}(\mathrm{t})$ are known with dynamic $\mathrm{CT}$, so using the deconvolution method $\mathrm{F} \cdot \mathrm{R}(\mathrm{t})$ and its height can be calculated to determine the tumor blood flow [15].

$$
B F=\int_{0}^{t} \frac{C t(t)}{R(t)} d t
$$

The arterial input was determined by placing a region of interest (ROI) over the aorta and analyzing the CT attenuation change over the $40 \mathrm{~s}$ of perfusion acquisition. Using the P-CT software program, a time density curve was automatically generated for the arterial input. All data were processed into a map that represents the blood flow.

A gastroenterology surgeon who had at least 5 years of experience in gastroenterological radiological imaging subsequently obtained ROI of the whole tumor, drawn freehand along the peripheral margin for computation of the perfusion parameters for each of the 4 sequential slices. The blood flow values were calculated in the workstation and a functional map was displayed in colors ranging from blue to red, with red being the higher range of display for blood flow (fig. 1). The blood flow values within the hand drawn ROI area were then averaged across the 4 slices. These 4 slices were selected carefully to include the tumor where largest in diameter, so the blood flow representing the whole tumor would be averaged out from the largest area possible.

\section{Histopathological Analysis}

Formalin-fixed paraffin-embedded tissues of the gastric cancer were sectioned at a thickness of $4 \mu \mathrm{m}$. Hematoxylin and eosin staining was performed to verify the morphologic diagnosis of gastric cancer and to determine the stage, histological grade and Lauren's classification.

Immunohistochemical studies were performed using a monoclonal antibody against CD34 protein, to highlight vessel walls. The tumor was scanned by using a conventional light microscope (BX50; Olympus, Tokyo, Japan) at low magnification $(40 \times)$ to search for the areas with strong CD34 staining. The stained areas were photographed at a magnification of $100 \times(10 \times$ objective lens and $10 \times$ ocular lens). The images were recorded by a chargecoupled device video camera (HC-300/OL; Olympus) mounted on top of the microscope and a color image freezer (Photograb-300 SH-3; Fujifilm, Tokyo, Japan). The acquired image files were opened using an image-processing software program (Adobe Photoshop 4.0J; Adobe Systems, San Jose, Calif., USA) to extract the dark brown stained vessels.

For a quantitative analysis of the extracellular matrix in cancer stroma, tissue collagen was highlighted by azan staining. As done in the CD34 staining, areas of the tumor with strong blue azan staining were chosen for extraction of the bright blue bands of collagen. To extract bright blue pixels from the RGB image, the other color pixels were erased repeatedly using a wand tool.

Every image was loaded into a public domain image-processing program (ImageJ; National Institutes of Health, available at http://rsb.info.nih.gov/ij/download.html) to calculate the pixel area of the stained areas. This procedure was performed by two investigators on 10 representative fields with the highest amount of vessels and collagen and the mean values were used for each tumor. CD34 staining was used to quantify areas of microvessels and azan staining for tumor stroma. The amount of microvessel and tumor stroma was expressed as the percentage pixels of the total pixel area of the image and the mean percentage of the 10 areas was used as microvessel density and tumor stromal density (fig. 2).

Tumor characteristics (size, location, TNM stage) were recorded. The blood flow was compared with the histopathological characteristics of gastric cancer (histological grade, Lauren's classification) and then it was evaluated for any correlations with microvessel density and tumor stromal density.

\section{Statistical Analysis}

All data were collected and analyzed retrospectively. Statistical analysis was performed using the Mann-Whitney $U$ test for comparisons of the continuous datasets and $\mathrm{p}$ values were calculated for each comparison. The data are expressed as the median and range. Correlations between the blood flow and microvessel density, tumor stromal density were analyzed using the Spearman's rank correlation test. A p value $\leq 0.05$ was considered to indicate a statistically significant difference. All statistical analyses were performed using StatView-J Version 5.0 (SAS Institute, Inc., Cary, N.C., USA).

\section{Results}

None of the specimens possessed any necrotic tissue. No significant correlation was found between blood flow and the tumor location, macroscopic appearance, or size (table 1).

In the cases without distant metastases or peritoneal dissemination, there was a significant decrease in blood flow in T3/T4, in comparison to T2. There was no difference in lymph node status or distant metastases, of which both were liver metastases, but patients with peritoneal dissemination had a lower blood flow in comparison to those without. The advanced cases according to TNM staging had lower blood flow than the earlier staged cases. According to the histological grading, gastric cancers are typically classified as either differentiated or undifferentiated. Of the 50 cases, 20 were well to moderately differentiated and the remaining 30 were classified as undifferentiated. The undifferentiated type of gastric cancer was found to have lower blood flow in comparison to the differentiated type.

Of the 40 surgical specimens with Lauren's diffuse type carcinomas, 11 were found to have decreased blood flow in comparison to the 29 mixed or intestinal type (table 2). As for the stromal structure, when compared among the different blood flows, there was no significant correlation with the microvessel density. However, blood flow significantly decreased with increased tumor stromal density (fig. 3). 

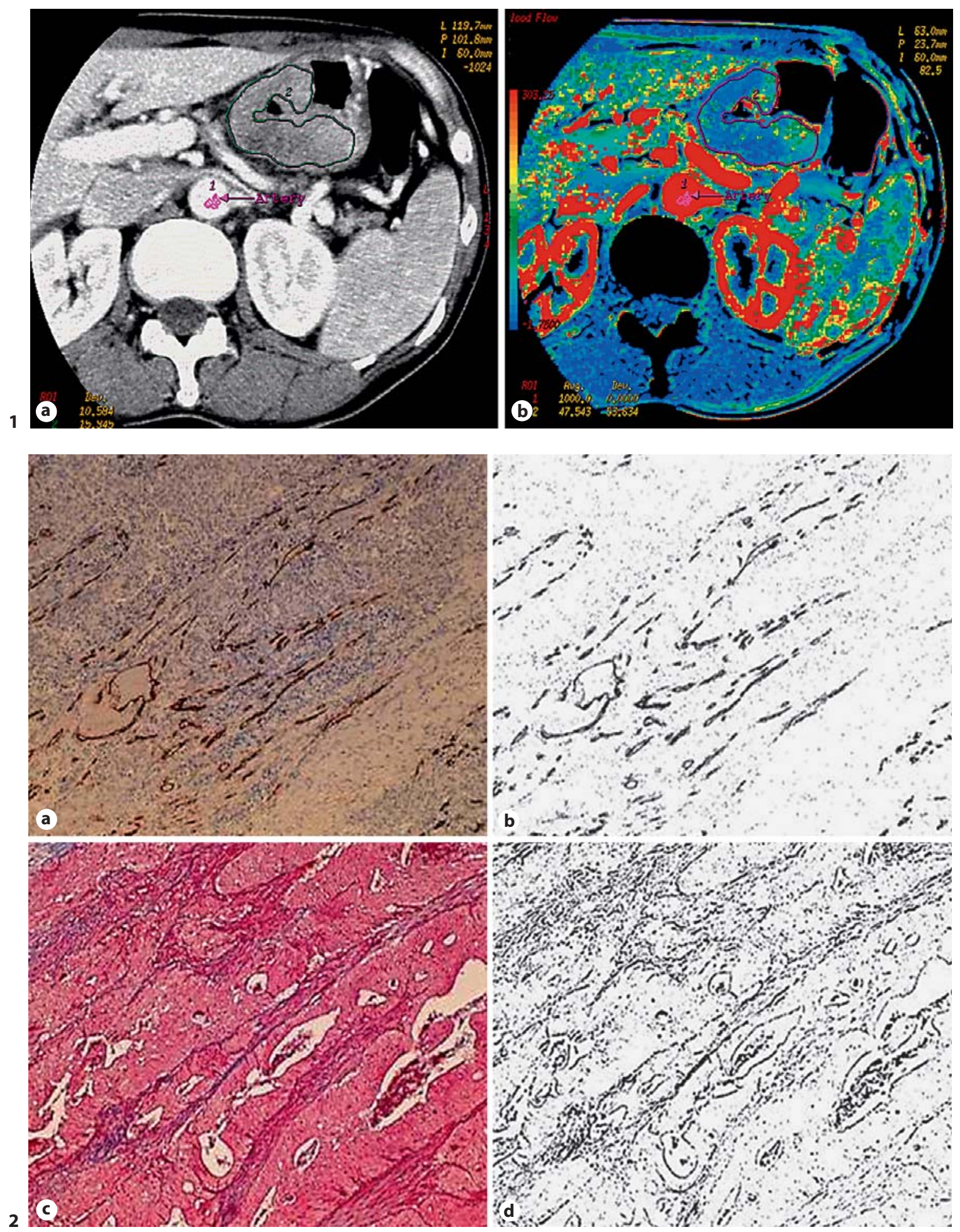

Fig. 1. P-CT images of gastric cancer. a CT image shows ROI placed within the tumor. b Perfusion map of blood flow shows the outlined area of the tumor to be relatively blue, with a fairly low perfusion, an average blood flow of $49.8 \mathrm{ml} / \mathrm{min} / 100 \mathrm{~g}$.
Fig. 2. CD34 and azan staining of tumor specimens $(\times 100)$. a CD34 staining highlights the vessel walls. b With an imageprocessing software, the stained areas were extracted to calculate microvessel density. c Azan staining highlighted stromal collagen in blue. d The stained stroma areas were quantified to calculate tumor stromal density. 
Table 1. Correlation between tumor location, appearance, size and blood flow

\begin{tabular}{lll}
\hline Variables & Cases & $\mathrm{BF}, \mathrm{ml} / \mathrm{min} / 100 \mathrm{~g}$ \\
\hline $\begin{array}{l}\text { Location } \\
\quad \text { Upper }\end{array}$ & 12 & $50.2(22.8-124)$ \\
$\quad$ Middle & 21 & $29.4(13.2-73.7)$ \\
$\quad$ Lower & 17 & $40.7(17.8-138)$ \\
Macroscopic appearance & & \\
$\quad$ Borrman type 1 & 2 & $87.1(50.7-124)$ \\
$\quad$ Borrman type 2 & 10 & $38.8(22.2-107)$ \\
$\quad$ Borrman type 3 & 22 & $28.1(17.4-138)$ \\
$\quad$ Borrman type 4 & 16 & $38.8(13.2-104)$ \\
Tumor size & & \\
$\quad \sim 5 \mathrm{~cm}$ & 10 & $49.1(22.2-90.1)$ \\
$\quad 5-10 \mathrm{~cm}$ & 17 & $48.3(21.5-138)$ \\
$>10 \mathrm{~cm}$ & 13 & $38.7(13.2-107)$ \\
\hline
\end{tabular}

Blood flow is noted as median (range). Location and macroscopic appearance were based on preoperative radiological examinations. Tumor size was recorded in resected surgical specimens, and inoperable cases were excluded.

Table 2. Correlation between tumor characteristics and blood flow

\begin{tabular}{lccc}
\hline Variables & Cases & BF, ml/min/100 g & p value \\
\hline $\begin{array}{l}\text { Tumor depth } \\
\quad \text { T2 }\end{array}$ & 12 & $65.1(23.7-13.2)$ & \\
$\quad$ T3/T4 & 19 & $39.0(13.2-138)$ & 0.0285 \\
$\begin{array}{l}\text { Nodal status } \\
\quad \text { Negative }\end{array}$ & 9 & $52.0(23.3-138)$ & \\
$\quad$ Positive & 22 & $39.9(13.2-113)$ & 0.3169 \\
$\begin{array}{l}\text { Distant metastases } \\
\quad \text { None }\end{array}$ & 48 & $38.8(13.2-138)$ & \\
$\quad$ Present & 2 & $27.0(25.9-28.0)$ & 0.2761 \\
$\begin{array}{l}\text { Peritoneal dissemination } \\
\quad \text { None }\end{array}$ & 31 & $43.1(13.2-138)$ & \\
$\quad$ Present & 19 & $29.4(17.4-104)$ & 0.0376 \\
$\begin{array}{l}\text { TNM stage } \\
\quad \text { Ib/II }\end{array}$ & 13 & $52.0(23.3-138)$ & \\
$\quad$ III/IV & 37 & $36.9(13.2-104)$ & 0.0196 \\
Histological grade & & & \\
$\quad \begin{array}{l}\text { Differentiated } \\
\quad \text { Undifferentiated }\end{array}$ & 20 & $56.1(17.4-138)$ & 0.0003 \\
Lauren's classification & 30 & $29.7(13.2-104)$ & \\
$\quad$ Mixed/intestinal & 29 & $50.7(21.5-138)$ & 0.0125 \\
$\quad$ Diffuse & 11 & $35.0(13.2-48.3)$ & \\
\hline
\end{tabular}

Blood flow is noted as median (range).

\section{Discussion}

The advent of multidetector CT techniques has led to an accurate assessment of staging for gastric cancer [16] along with other preoperative staging factors. CT imaging may also be useful in the assessment of therapeutic effects for chemotherapy or radiation therapy. Because many anti-angiogenetic agents are not cytotoxic but instead produce disease stabilization, the measurement of tumor size alone may be non-informative regarding the therapeutic effect [17]. Attention has centered on the use of physiologic imaging techniques and led to the development of P-CT. $\mathrm{P}-\mathrm{CT}$ is able to calculate the parameters associated with tissue perfusion, such as blood flow, blood volume, mean transit time and permeability. This study used a single parameter to assess perfusion and chose blood flow for its fairly simple relationship to the changes in CT units, in comparison to the other parameters.

The clinical utility of this technique has been initially established in the central nervous system and now is regarded as one of the most sensitive sequences for acute cerebral ischemia. In malignant neoplasms, the ability to assess tumor perfusion is potentially useful in understanding the tissue microenvironment, especially with regard to the vascularity and stroma. There are a number of reports in the past that have shown the dynamics of contrast enhancement can reflect the microenvironment. Dugdale et al. [18] reported a correlation between increased tumor blood flows with the higher grade of lymphoma. Another study on cervical cancer observed a correlation between oxygen pressure and tumor blood flow [19]. With the current study, it was possible to visualize the decrease in tumor perfusion with the increasing level of malignancy in terms of several clinicopathological factors.

Despite the significant correlation between blood flow and tumor depth, peritoneal dissemination and stage, there was no significant difference when patients with and without lymph node metastasis were compared. A report on colorectal cancer also failed to show a relationship between perfusion parameters and lymph node metastasis [20]. The reason for this may be that tumor perfusion is closely related to vascular invasion and hematogenous spreading rather than lymphatic metastasis [21]. Our study included 2 patients with hepatic metastasis, and although we could not see any significance in the decrease with such few cases, both patients had an extremely low blood flow average of 27.0.

Tumor grade refers to the degree of differentiation of tumor cells and has been shown to correlate with the ag- 
Fig. 3. Scatter plots of microvessel density and tumor stromal density against blood flow. a There was no significant correlation between the microvessel density and blood flow $(\mathrm{R}=0.261, \mathrm{p}=0.266)$. b A strong correlation was seen between tumor stromal density and blood flow $(\mathrm{R}=$ $0.861, \mathrm{p}<0.0001)$.

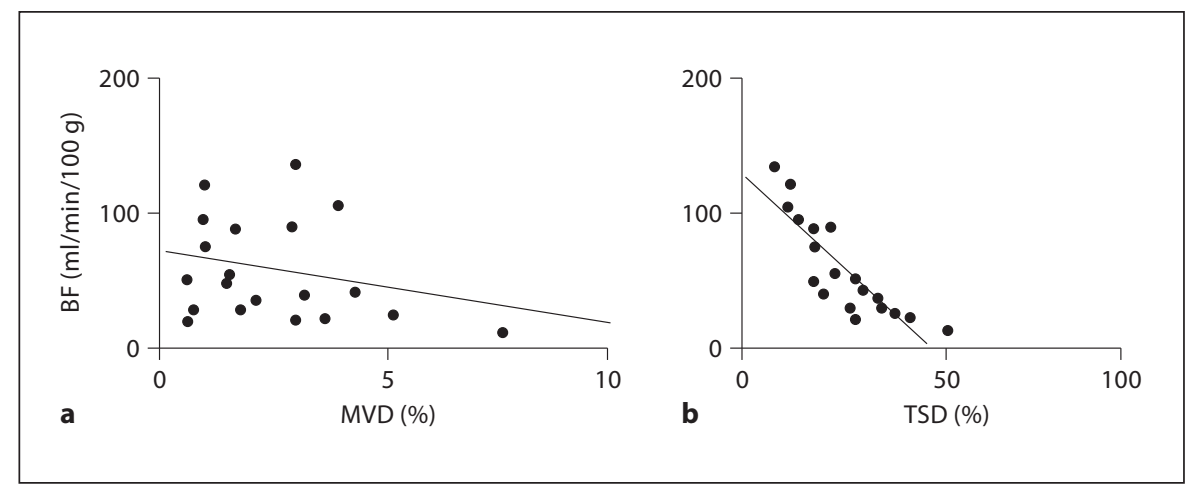

gressiveness of the neoplasm [22]. The prognostic impact of histological grading in gastric cancer has been well documented and has indicated poor prognosis in patients with the undifferentiated type [1]. In the current study, undifferentiated gastric carcinoma had lower blood flow in comparison with the differentiated type, suggesting that tumor perfusion, reflecting differentiation, may be associated with the malignant character of gastric cancer.

Lauren's classification divides gastric cancer into two major histologic types, namely intestinal or diffuse. This system describes tumors on the basis of microscopic configuration and growth pattern. Diffuse type gastric cancer, the main part of which is undifferentiated carcinoma, displays a diffusely invasive growth pattern. In comparison to intestinal type gastric cancers, this type of cancer is associated with a worse prognosis [23]. Our study shows the diffuse type gastric cancers to have decreased blood flow in comparison to the intestinal type, suggesting that blood flow may be useful in evaluating the natural history of gastric carcinoma.

Furthermore, diffuse type gastric cancers are difficult to be diagnosed preoperatively. The reliability of a preoperative assessment of this aggressive type of cancer is important with regard to the surgical strategy, especially the extent of resection. The diagnosis of the diffuse type is not always accurate with an endoscopic biopsy [24], and blood flow assessed with P-CT may be useful in preoperative assessment.

The development of a tumor blood supply through the process of angiogenesis is essential for the growth and metastasis of tumors [5]. Microvessel density is currently applied as an indicator for tumor angiogenetic activity [25]. However, changes in microvessel density do not always correspond with the changes in tissue perfusion because they are affected by mature vessels as well as immature and thin vessels independent of blood flow, in- duced by tumor angiogenesis [26, 27]. In this study, microvessel density did not have any relationship with blood flow, despite the fact that tumor stromal density had a significant correlation.

After the administration of the iodinated contrast medium, the immediate tumor enhancement is largely due to the intravascular contrast medium. As time progresses, leakage of the contrast medium into the extravascular space continues and reaches equilibrium [28]. In P-CT, the contrast medium in both the intra- and extravascular space yielding a rapid series of images is used to assess perfusion. The blood flow assessed with perfusion imaging reflects only the functional vessels with a lumen, and not the functionless tumor vascularity [29]. When the volume of the contrast medium administered is the same, the enhancement of the tumor depends on the tumor blood flow, which is also affected by the pressure of the extravascular stroma. Intratumoral interstitial hypertension, which leads to collapse of the vessels and reduction of perfusion, is increased with the development of tumor vasculature [30,31], and this is consistent with the current results where an increased stromal density resulted in a decreased flow. There are still no published studies on the P-CT parameters in comparison to the stromal density.

Another reason microvessel density is an inadequate marker for tissue perfusion is that this marker has only been studied immunohistochemically in vitro using surgical specimens. Other less invasive methods, such as biopsy specimens taken endoscopically, are located on the surface of the cancer tissue and do not represent the microenvironment of the whole tumor. The ability to determine tumor perfusion in vivo by using imaging is valuable when surgical specimens are not available, as in inoperable cases. Blood flow calculated with P-CT could be an in vivo marker of tumor perfusion. 
P-CT may enable risk stratification for patients with cancer with the potential to individualize the patient's treatment by matching the therapy to the tumor's biological character. Patients with aggressive tumors such as scirrhous type gastric cancer may be suitable for additional treatment or invasive local treatments could be withheld when unlikely to be of benefit. However, larger studies are needed to confirm our results.

There were several limitations in this study. First, there was a selection bias, patients with gastric cancer too small to be detected on CT were excluded. Recent techniques in multiplanar reformatting may improve the visualization of each tumor, and shortened scanning time with multidetector row $\mathrm{CT}$ may allow visualization of smaller lesions. When this becomes possible, more early staged cancers could be included in this study with increased accuracy.

Second, the limited view of tumors afforded by four section repeated imaging may lead to the misinterpretation of blood flow values of the tumor as a whole. We took great care in the manual drawing of ROIs along the tumor, and the blood flow is averaged out within the defined tumor margin, to avoid evaluation of only a limited section of the organ. New multidetector row CT equipment with wider detectors may permit larger areas of the tumor to be included in the scan, allowing a more precise evaluation of perfusion parameters.

Third, no validation studies were performed for the P-CT parameters, such as comparing blood flow with direct measurement of blood flow with a sonogram. This study was a preliminary study, and our purpose was not the measurement of tumoral blood flow, but the evaluation of the correlation between the blood flows calculated with this specific software and the clinicopathological features of the tumor. This study supports blood flow as a potential surrogate marker for angiogenesis.

In addition, the radiation dose delivered to each patient with the P-CT technique was not assessed. The study was performed along with a routine diagnostic study and the patient would be exposed to an additional dose of radiation. More sophisticated CT technologies may allow reductions in the radiation in future studies.

\section{Conclusions}

In this study, there was a significant correlation between the tumor blood flow and tumor depth, peritoneal dissemination, TNM staging, histological grade, Lauren's classification and tumor stromal density. Tumor perfusion decreased as the stage and malignant character of the tumor advanced, thus the analysis of blood flow with P-CT may be helpful for achieving a better estimation of the individual survival and the identification of patients with a high risk of recurrence.

\section{Acknowledgments}

I would like to express my gratitude to my supervisor, Dr. Kiyohiko Shuto, whose expertise, understanding, and patience, added considerably to completing this research. I appreciate his vast knowledge and skill in many areas such as radiology, surgery and pathology, and his assistance in putting numerous presentations together. I would like to thank the other members of my research laboratory for the assistance they provided to get the research project rolling. In conclusion, I recognize that this research would not have been possible without the technological assistance provided by Mr. Noriyuki Yanagawa, along with his support in gathering all the material.

\section{References}

1 Crawford J: The gastrointestinal tract; in Robbins S, Kumar V, Schoen F (eds): Pathologic Basis of Disease. Philadelphia, Saunders, 1994, pp 755-783.

-2 Bozzetti F, Marubini E, Bonfanti G, Miceli R, Piano C, Gennari L: Subtotal versus total gastrectomy for gastric cancer: five-year survival rates in a multicenter randomized Italian trial. Italian Gastrointestinal Tumor Study Group. Ann Surg 1999;230:170-178.

-3 Roder JD, Bottcher K, Siewert JR, Busch R, Hermanek P, Meyer HJ: Prognostic factors in gastric carcinoma. Results of the German Gastric Carcinoma Study 1992. Cancer 1993; 72:2089-2097.

\footnotetext{
4 Siewert JR, Bottcher K, Stein HJ, Roder JD Relevant prognostic factors in gastric cancer: ten-year results of the German Gastric Cancer Study. Ann Surg 1998;228:449-461.

5 Folkman J: What is the evidence that tumors are angiogenesis dependent? J Natl Cancer Inst 1990;82:4-6.

6 Hurwitz H, Fehrenbacher L, Novotny W, Cartwright T, Hainsworth J, Heim W, Berlin J, Baron A, Griffing S, Holmgren E, Ferrara N, Fyfe G, Rogers B, Ross R, Kabbinavar F: Bevacizumab plus irinotecan, fluorouracil, and leucovorin for metastatic colorectal cancer. N Engl J Med 2004;350:2335-2342.
}

\footnotetext{
7 Yang JC, Haworth L, Sherry RM, Hwu P, Schwartzentruber DJ, Topalian SL, Steinberg SM, Chen HX, Rosenberg SA: A randomized trial of bevacizumab, an antivascular endothelial growth factor antibody, for metastatic renal cancer. N Engl J Med 2003; 349:427-434.

$\checkmark 8$ Chung YS, Maeda K, Sowa M: Prognostic value of angiogenesis in gastrointestinal tumours. Eur J Cancer 1996;32A:2501-2505.

-9 Hansen S, Grabau DA, Sorensen FB, Bak M, Vach W, Rose C: Vascular grading of angiogenesis: prognostic significance in breast cancer. Br J Cancer 2000;82:339-347.
} 
10 Matsuyama K, Chiba Y, Sasaki M, Tanaka H, Muraoka R, Tanigawa N: Tumor angiogenesis as a prognostic marker in operable nonsmall cell lung cancer. Ann Thorac Surg 1998;65:1405-1409.

-11 Cuenod CA, Fournier L, Balvay D, Guinebretiere JM: Tumor angiogenesis: pathophysiology and implications for contrast-enhanced MRI and CT assessment. Abdom Imaging 2006;31:188-193.

-12 Lee TY, Purdie TG, Stewart E: CT imaging of angiogenesis. Q J Nucl Med 2003;47:171-187.

$\checkmark 13$ Swensen SJ, Viggiano RW, Midthun DE, Muller NL, Sherrick A, Yamashita K, Naidich DP, Patz EF, Hartman TE, Muhm JR, Weaver AL: Lung nodule enhancement at CT: multicenter study. Radiology 2000;214: 73-80.

14 Sobin LH, Fleming ID: TNM Classification of Malignant Tumors, ed 5 (1997). Union Internationale Contre le Cancer and the American Joint Committee on Cancer. Cancer 1997;80:1803-1804.

15 Meier P, Zierler KL: On the theory of the indicator-dilution method for measurement of blood flow and volume. J Appl Physiol 1954; 6:731-744.

-16 Kumano S, Murakami T, Kim T, Hori M, Iannaccone R, Nakata S, Onishi H, Osuga K, Tomoda K, Catalano C, Nakamura H: T staging of gastric cancer: role of multi-detector row CT. Radiology 2005;237:961-966.
7 Kan Z, Phongkitkarun S, Kobayashi S, Tang Y, Ellis LM, Lee TY, Charnsangavej C: Functional CT for quantifying tumor perfusion in antiangiogenic therapy in a rat model. Radiology 2005;237:151-158.

18 Dugdale PE, Miles KA, Bunce I, Kelley BB, Leggett DA: CT measurement of perfusion and permeability within lymphoma masses and its ability to assess grade, activity, and chemotherapeutic response. J Comput Assist Tomogr 1999;23:540-547.

19 Haider MA, Milosevic M, Fyles A, Sitartchouk I, Yeung I, Henderson E, Lockwood G Lee TY, Roberts TP: Assessment of the tumor microenvironment in cervix cancer using dynamic contrast enhanced CT, interstitial fluid pressure and oxygen measurements. Int J Radiat Oncol Biol Phys 2005;62:11001107.

20 Li ZP, Meng QF, Sun CH, Xu DS, Fan M Yang XF, Chen DY: Tumor angiogenesis and dynamic CT in colorectal carcinoma: radiologic-pathologic correlation. World J Gastroenterol 2005;11:1287-1291.

21 Chen CN, Cheng YM, Lin MT, Hsieh FJ, Lee $\mathrm{PH}$, Chang KJ: Association of color Doppler vascularity index and microvessel density with survival in patients with gastric cancer Ann Surg 2002;235:512-518.

22 Adachi Y, Yasuda K, Inomata M, Sato K, Shiraishi N, Kitano S: Pathology and prognosis of gastric carcinoma: well versus poorly differentiated type. Cancer 2000;89:1418-1424.

23 Lauren P: The two histological main types of gastric carcinoma: diffuse and so-called intestinal-type carcinoma. an attempt at a histo-clinical classification. Acta Pathol Microbiol Scand 1965;64:31-49.
24 Jonasson L, Hallgrimsson J, Olafsdottir G: Gastric carcinoma: correlation of diagnosis based on biopsies and resection specimens with reference to the Lauren classification. APMIS 1994;102:711-715.

25 Weidner N: Intratumor microvessel density as a prognostic factor in cancer. Am J Pathol 1995;147:9-19.

26 Brown JM, Giaccia AJ: The unique physiology of solid tumors: opportunities (and problems) for cancer therapy. Cancer Res 1998;58:1408-1416.

27 Jain RK: Barriers to drug delivery in solid tumors. Sci Am 1994;271:58-65.

$>28$ Miles KA: Tumour angiogenesis and its relation to contrast enhancement on computed tomography: a review. Eur J Radiol 1999;30: 198-205.

-29 Aronen HJ, Gazit IE, Louis DN, Buchbinder BR, Pardo FS, Weisskoff RM, Harsh GR, Cosgrove GR, Halpern EF, Hochberg FH, et al: Cerebral blood volume maps of gliomas: comparison with tumor grade and histologic findings. Radiology 1994;191:41-51.

30 Boucher Y, Leunig M, Jain RK: Tumor angiogenesis and interstitial hypertension. Cancer Res 1996;56:4264-4266.

-31 Milosevic MF, Fyles AW, Hill RP: The relationship between elevated interstitial fluid pressure and blood flow in tumors: a bioengineering analysis. Int J Radiat Oncol Biol Phys 1999;43:1111-1123. 